\title{
Purity and Correlation of a Cavity Field Interacting with a SC Charge Qubit with a Lossy Cavity
}

\author{
M. Sebawe Abdalla • A.-S.F. Obada • \\ A.-B.A. Mohamed · E.M. Khalil
}

Received: 1 May 2013 / Accepted: 8 October 2013 / Published online: 27 November 2013

(c) The Author(s) 2013. This article is published with open access at Springerlink.com

\begin{abstract}
A single-mode microwave cavity field, coupled to its reservoir, interacting generally with a superconducting charge qubit is considered. Using a certain canonical transformation for the qubit states, the system is transformed into the usual Jaynes-Cummings model. The solution of the master equation of this system, in the case of a high- $Q$ cavity is obtained. The temporal evolution of the population inversion is explored. The effects of cavity damping on the purity of the qubit, the field and the global system state are studied. It is found that due to the coupling between the system and environment, the purity is lost. The entanglement is compared with total correlation. It is found that, with the damping parameter, the asymptotic value of the correlation measure is not null, since the global system evolves to a classically correlated state. The negativity is used as an indicator of the degree of entanglement between the qubit and the field. The results indicate the sensitivity of these aspects to change of the damping parameter.
\end{abstract}

Keywords Charge qubit · Purity · Correlation

\section{Introduction}

Superconducting qubits have been considered as possible candidates for quantum information processing and have attracted much attention in recent years [1-8]. It has been experimentally demonstrated that the superconducting qubits possess macroscopic quantum

M. Sebawe Abdalla

Mathematics Department, College of Science, King Saud University P.O. Box 2455, Riyadh 11451,

Saudi Arabia

A.-S.F. Obada · E.M. Khalil ( $\varangle)$

Mathematics Department, Faculty of Science, Al-Azher University, Nassr City 11884, Cairo, Egypt

e-mail: eiedkhalil@yahoo.com

A.-B.A. Mohamed

Mathematics Department, Faculty of Science, Assiut University, Assiut, Egypt 
coherence and can be used to realize the conditional two-qubit gate, where the superconducting qubits have gained substantial interest as devices for application in quantum information processing [1, 2, 9]. Here, Josephson qubits are recognized as being among the most promising devices to implement solid state quantum computation [10]. The manipulation of quantum states in individual and coupled qubits (Cooper-pair box) has been demonstrated experimentally [11] and the behavior of charge oscillations in superconducting Cooper pair boxes weakly interacting with an environment has been discussed [12]. Superconducting circuits can behave like virtual atoms and test quantum mechanics at macroscopic scales and can be used to conduct atomic-physics experiments on a silicon chip [9]. Furthermore, the quantum dynamics of a Cooper-pair box with a superconducting loop in the presence of a non-classical microwave field have been investigated [13]. The development in the theory of quantum information and the correlations are playing an increasingly fundamental and important role in the study and exploitation of quantum advantages. In particular, it has been widely recognized, during the past few decades, that correlations (including entanglement, and more generally, quantum correlations) are valuable resources for quantum information processing [14-18].

The study of entanglement dynamics is crucial for the realization of quantum algorithms and quantum information processing protocols [19]. On the other hand, realistic quantum systems will inevitably interact with the environments. The interaction between the system and the environment usually leads to a decoherence process [20,21]. This is a fundamental obstacle to perform reliable quantum computation. Therefore, a number of studies have been devoted to study the dynamics of quantum entanglement under environmental effects [22]. To measure the entanglement (purity loss) and classical correlation, there are more than one measure in the literature, such as the von Neumann reduced entropy or linear entropy, the negative mutual entropy [23], the negative mutual information (index of classical correlation) [24], the sum of negative eigenvalues of the partially transposed density matrix [25]. But the total correlation in a bipartite quantum system can be measured by quantum mutual information [26, 27], which may be divided into classical and quantum parts [28-30].

This contribution is organized as follows. Section 2 is devoted to introduce the system Hamiltonian model and introduce an exact solution of the master equation in the case of a high- $Q$ cavity for a general interaction of a single-mode microwave cavity field with a superconducting charge qubit. In Sect. 3 we discuss the population inversion, while in Sect. 4 we investigate the purity of the system and its subsystems state, by using linear entropy. The entanglement and the total correlation are discussed by using the negativity in Sect. 5. Our conclusion is given in Sect. 6.

\section{The Physical Model and Its Solution}

We consider a system consisting of a single-mode microwave cavity field interacting with a superconducting charge qubit. The Hamiltonian for this system is a generalization of $[31,32]$. This Hamiltonian can be written as:

$$
\frac{\hat{H}}{\hbar}=\omega \hat{a}^{\dagger} \hat{a}+E_{z} \hat{\sigma}_{z}-E_{J}\left[\hat{\sigma}_{+} e^{\frac{i \pi}{\Phi_{0}}\left(\hat{I} \Phi_{C}+\eta \hat{a}+\eta^{*} \hat{a}^{\dagger}\right)}+h c\right],
$$

where $\omega$ is the frequency of the cavity field. The operators $\hat{\sigma}_{z}$ and $\hat{\sigma}_{ \pm}$are defined by $\hat{\sigma}_{z}=$ $|e\rangle\langle e|-| g\rangle\langle g|$ and $\hat{\sigma}_{+}=|e\rangle\left\langle g\left|, \hat{\sigma}_{-}=\right| g\right\rangle\langle e|$, where $|e\rangle$ and $|g\rangle$ are the excited and ground states of the qubit, respectively. Here, $a$ and $a^{\dagger}$ are annihilation and creation operators of 
the cavity and $\hat{I}$ is an identity operator, $E_{J}$ is the coupling constant of the interaction of the atom with the cavity. The qubit charging energy $E_{z}=-2 E_{c h}\left(1-2 n_{g}\right)$, which depends on the gate charge $n_{g}$. The single-electron charging energy is $E_{c h}=\frac{e^{2}}{2\left(C_{g}+2 C_{J}\right)}, C_{g}$ and $C_{J}$ are the Josephson junction and the gate capacities. The third term is the nonlinear charge qubitphoton interaction, where $\Phi_{C}$ the flux is generated by a classical applied magnetic field and $\Phi_{0}$ is the quantum flux. The parameter $\eta$ has units of magnetic flux and its absolute value represents the strength of the quantum flux inside the cavity and can be expressed as $|\eta|=S$ $\sqrt{\frac{\omega \hbar}{\varepsilon_{0} V C^{2}}}\left|\cos \left(\frac{2 \pi z_{0}}{g}\right)\right|[31,32]$, which shows that $|\eta|$ depends on the area $S$ of the surface defined by the contour of the superconducting quantum interference device (SQUID) and its position $z_{0}$, the wavelength $g$ of cavity field, and the volume $V$ of the cavity. If the light field is not so strong (e.g., the average number of photons inside the cavity $N=\hat{a}^{\dagger} \hat{a} \leq 100$ ), then we can only keep the first order of $\frac{\pi \eta}{\Phi_{0}}$ and safely neglect all higher orders. Thus, the Hamiltonian (1) becomes

$$
\begin{aligned}
\frac{\hat{H}}{\hbar}= & \omega \hat{a}^{\dagger} \hat{a}+E_{z} \hat{\sigma}_{z}-E_{J}\left(\hat{\sigma}_{x} \cos \frac{\pi \Phi_{C}}{\Phi_{0}}+\hat{\sigma}_{y} \sin \frac{\pi \Phi_{C}}{\Phi_{0}}\right) \\
& -\frac{\pi E_{J}}{\Phi_{0}}\left(-\hat{\sigma}_{x} \sin \frac{\pi \Phi_{C}}{\Phi_{0}}+\hat{\sigma}_{y} \cos \frac{\pi \Phi_{C}}{\Phi_{0}}\right)\left(\eta \hat{a}+\eta^{*} \hat{a}^{\dagger}\right) .
\end{aligned}
$$

From the above equation we can get all the information related to the well known JaynesCummings model JCM. We assume that the qubit-photon system works at low temperatures $T$ (e.g., $T=30 \mathrm{mK}$ in Refs. [33-35]), then the mean number of thermal photons in the cavity is almost negligible in the microwave regime [31,32], and the cavity is approximately considered in the zero-temperature environment. Therefore, in this paper we take into account only the field mode damping and ignore the qubit state damping, while the cavity is assumed to be at zero temperature. Then, the master equation for the density matrix of the combined (qubit-field) system is given by:

$$
\frac{d \hat{\rho}(t)}{d t}=-i[\hat{H}, \hat{\rho}(t)]+\gamma\left(\left[a \hat{\rho}(t), \hat{a}^{\dagger}\right]+\left[\hat{a}, \hat{\rho}(t) \hat{a}^{\dagger}\right]\right),
$$

where $\gamma$ is the cavity field damping parameter. To solve the master equation (3), we make the following transformations of the atomic operators

$$
\begin{aligned}
& \hat{S}_{x}=\left(\hat{\sigma}_{x} \cos \frac{\pi \Phi_{C}}{\Phi_{0}}+\hat{\sigma}_{y} \sin \frac{\pi \Phi_{C}}{\Phi_{0}}\right) \\
& \hat{S}_{y}=\left(-\hat{\sigma}_{x} \sin \frac{\pi \Phi_{C}}{\Phi_{0}}+\hat{\sigma}_{y} \cos \frac{\pi \Phi_{C}}{\Phi_{0}}\right) \\
& \hat{S}_{z}=\hat{\sigma}_{z}
\end{aligned}
$$

These rotated operators $\hat{S}_{z}, \hat{S}_{x}$ and $\hat{S}_{y}$ satisfy the following properties

$$
\left[\hat{S}_{x}, \hat{S}_{y}\right]=2 i \hat{S}_{z}, \quad\left[\hat{S}_{y}, \hat{S}_{z}\right]=2 i \hat{S}_{x}, \quad\left[\hat{S}_{z}, \hat{S}_{x}\right]=2 i \hat{S}_{y}
$$

The eigenstates of $\hat{S}_{z}$ is the same eigenstates of $\hat{\sigma}_{z}$. Thus, the Hamiltonian (2) becomes

$$
\frac{\hat{H}}{\hbar}=\omega \hat{a}^{\dagger} \hat{a}+E_{z} \hat{S}_{z}-E_{J} \hat{S}_{x}-\frac{\pi E_{J}}{\Phi_{0}} \hat{S}_{y}\left(\eta \hat{a}+\eta^{*} \hat{a}^{\dagger}\right)
$$


To simplify this Hamiltonian, we define the transformation between the states $|e\rangle,|g\rangle$ and the states $|\uparrow\rangle,|\downarrow\rangle$ which take the following form:

$$
|\uparrow\rangle=\cos \xi|e\rangle+\sin \xi|g\rangle, \quad|\downarrow\rangle=\cos \xi|g\rangle-\sin \xi|e\rangle, \quad \xi=\frac{1}{2} \tan ^{-1}\left(\frac{E_{J}}{E_{z}}\right) .
$$

The rotated operators $\hat{Q}_{z}$ and $\hat{Q}_{ \pm}$defined as $\hat{Q}_{+}=|\uparrow\rangle\left\langle\downarrow\left|, \hat{Q}_{-}=\right| \downarrow\right\rangle\langle\uparrow|$ and $\hat{Q}_{z}=$ $|\uparrow\rangle\langle\uparrow|-| \downarrow\rangle\langle\downarrow|$, satisfy the following properties

$$
\left[\hat{Q}_{+}, \hat{Q}_{-}\right]=\hat{Q}_{z}, \quad\left[\hat{Q}_{z}, \hat{Q}_{ \pm}\right]= \pm 2 \hat{Q}_{ \pm}
$$

This in fact would give us an advantage to transform the Hamiltonian (6) after applying the rotated wave approximation into the form

$$
\begin{aligned}
\hat{\tilde{H}} & =\omega \hat{a}^{\dagger} \hat{a}+\Omega_{0} \hat{Q}_{z}-\frac{\pi E_{J}}{\Phi_{0}} \cos ^{2} \xi\left(\eta \hat{a} \hat{Q}_{+}+\hat{Q}_{-} \eta^{*} \hat{a}^{\dagger}\right), \\
\Omega_{0} & =\sqrt{E_{z}^{2}+\left(E_{J}\right)^{2}} .
\end{aligned}
$$

Thus we have managed to remove the driving term from the Hamiltonian (9) while its effect is concealed in the augmented atomic frequency $\Omega_{0}$, which shifts the atomic energy levels to $\Omega_{0}= \pm \sqrt{E_{z}^{2}+\left(E_{J}\right)^{2}}$. The connections between the original operators $\hat{\sigma}^{\prime} s$ and the rotating operators $\hat{Q}^{\prime} s$ take the following form

$$
\begin{aligned}
& \hat{\sigma}_{x}=\cos \frac{\pi \Phi_{C}}{\Phi_{0}}\left(\cos 2 \xi \hat{Q}_{x}-\sin 2 \xi \hat{Q}_{z}\right)-\sin \frac{\pi \Phi_{C}}{\Phi_{0}} \hat{Q}_{y} \\
& \hat{\sigma}_{y}=\sin \frac{\pi \Phi_{C}}{\Phi_{0}}\left(\cos 2 \xi \hat{Q}_{x}-\sin 2 \xi \hat{Q}_{z}\right)+\cos \frac{\pi \Phi_{C}}{\Phi_{0}} \hat{Q}_{y} \\
& \hat{\sigma}_{z}=\left(\cos 2 \xi \hat{Q}_{z}+\sin 2 \xi \hat{Q}_{x}\right)
\end{aligned}
$$

Note here the mixing of these operators, which will show their effect when discussing the different phenomena later. After the above transforms, the master equation of the qubit-field system is given by:

$$
\frac{d \hat{\tilde{\rho}}(t)}{d t}=-i[\hat{\tilde{H}}, \hat{\tilde{\rho}}(t)]+\gamma\left(\left[a \hat{\tilde{\rho}}(t), \hat{a}^{\dagger}\right]+\left[\hat{a}, \hat{\tilde{\rho}}(t) \hat{a}^{\dagger}\right]\right) .
$$

The analytic methods for obtaining solutions of Eq. (11) in the case of a high- $Q$ cavity $(\gamma \ll \lambda)$, employ the so-called dressed-states representation [36-38], i.e. representation consisting of the complete set of Hamiltonian eigenstates. For lossless cavity the full set of dressed states are

$$
\begin{aligned}
& \left|\Psi_{n}^{ \pm}\right\rangle=\Phi_{n}^{ \pm}|\uparrow, n\rangle \pm \Phi_{n}^{\mp}|\downarrow, n+1\rangle \quad(n=0,1,2, \ldots), \\
& \Phi_{n}^{ \pm}=\frac{1}{\sqrt{2}} \sqrt{1 \mp \frac{\delta}{\eta_{n}}}, \quad \eta_{n}=\sqrt{\delta^{2}+v_{n}^{2}}, \quad v_{n}=\lambda \cos ^{2} \xi \sqrt{n+1}, \\
& \lambda=\frac{\pi|\eta| E_{J}}{\Phi_{0}}, \quad \delta=\frac{\Omega_{0}-\omega}{2}
\end{aligned}
$$


In an invariant subspace spanned by $|\uparrow, n\rangle$ and $|\downarrow, n+1\rangle$, the eigenvalues of $\hat{H}$ are given by:

$$
E_{n}^{ \pm}=\mu_{n} \pm \eta_{n}, \quad \mu_{n}=\omega\left(n+\frac{1}{2}\right)
$$

where

$$
\tilde{H}\left|\Psi_{n}^{ \pm}\right\rangle=E_{n}^{ \pm}\left|\Psi_{n}^{ \pm}\right\rangle, \quad \tilde{H}|\downarrow, 0\rangle=-\frac{\omega_{\circ}}{2}|\downarrow, 0\rangle .
$$

To solve Eq. (11) in the high- $Q$ limit, we express the annihilation operator $a$ and the photon number operator $\hat{a}^{\dagger} \hat{a}$ in terms of the dressed states. Then we use the representation $J(t)=$ $e^{i \hat{\tilde{H}} t} \rho(t) e^{-i \hat{\hat{H}} t}$ and obtain an equation for $J(t)$ in terms of the dressed states (12). The exact equation for $J(t)$ is found to be a sum of time-independent and time-dependent terms. The time-dependent terms in this equation oscillate at the frequency proportional to the qubitfield coupling $g$. It can be shown that the contribution of the oscillatory terms is of the order of $\left(\gamma^{2} / g^{2}\right)$. Hence, if we make the secular approximation [36-38], i.e, we neglect the oscillatory terms by assuming $(\gamma \ll g)$, with the representation

$$
\dot{J}(t)=e^{i \hat{\tilde{H}} t} \frac{\partial \hat{\rho}(t)}{\partial t} e^{-i \hat{\tilde{H}} t}+i[\hat{\tilde{H}}, J(t)]
$$

when we let that $\chi_{m n}^{i j}=\left\langle\Psi_{m}^{i}|J(t)| \Psi_{n}^{j}\right\rangle, i, j=+,-$, the off-diagonal elements of the density matrix $J(t)$ are given by

$$
\begin{array}{ll}
\chi_{m n}^{ \pm \pm}(t)=\chi_{m n}^{ \pm \pm}(0) e^{-\gamma\left(m+n+\Phi_{m}^{\mp 2}+\Phi_{n}^{\mp 2}\right) t}, & \forall m \neq n . \\
\chi_{m n}^{ \pm \mp}(t)=\chi_{m n}^{ \pm \mp}(0) e^{-\gamma\left(m+n+\Phi_{m}^{\mp 2}+\Phi_{n}^{ \pm 2}\right) t}, & \forall m, n .
\end{array}
$$

But the diagonal elements of density matrix $J(t)$ satisfy the equations:

$$
\dot{\chi}_{n, n}^{ \pm \pm}(t)=2 \gamma\left[f_{n+1}^{ \pm 2} \chi_{n+1, n+1}^{ \pm \pm}(t)+g_{n+1}^{ \pm 2} \chi_{n+1, n+1}^{\mp \mp}(t)-\left(n+\Phi_{n}^{\mp 2}\right) \chi_{n, n}^{ \pm \pm}(t)\right] .
$$

Where $f_{n}^{ \pm}=\sqrt{n} \Phi_{n-1}^{ \pm} \Phi_{n}^{ \pm}+\sqrt{n+1} \Phi_{n-1}^{\mp} \Phi_{n}^{\mp}$, and $g_{n}^{ \pm}=\sqrt{n} \Phi_{n-1}^{ \pm} \Phi_{n}^{\mp}-\sqrt{n+1} \Phi_{n-1}^{\mp} \Phi_{n}^{ \pm}$. The solution of the coupled differential equation (17), can be written as

$$
\begin{aligned}
\chi_{n, n}^{ \pm \pm}(t)= & e^{-2 \gamma\left(n+\Phi_{n}^{\mp 2}\right) t} \chi_{n, n}^{ \pm \pm}(0) \\
& +2 \gamma \int_{0}^{t} e^{2 \gamma\left(n+\Phi_{n}^{\mp 2}\right) \tau}\left(f_{n+1}^{ \pm 2} \chi_{n+1, n+1}^{ \pm \pm}(\tau)+g_{n+1}^{ \pm 2} \chi_{n+1, n+1}^{\mp \mp}(\tau)\right) d \tau .
\end{aligned}
$$

If we assume that there is an upper limit on the number of photons initially present in the system [37,38], so $\chi_{N+1, N+1}^{ \pm \pm}(t)=0$ is always true since the number of photons in the cavity will decrease only. Then at $n=N, N-1, \ldots$

$$
\begin{aligned}
\chi_{N, N}^{ \pm \pm}(t)= & e^{-2 \gamma\left(N+\Phi_{N}^{\mp 2}\right) t} \chi_{N, N}^{ \pm}(0), \\
\chi_{N-1, N-1}^{ \pm \pm}(t)= & e^{-2 \gamma\left(N+\Phi_{N-1}^{\mp 2}-1\right) t} \chi_{N-1, N-1}^{ \pm \pm}(0) \\
& +\frac{f_{N}^{ \pm 2} \chi_{N, N}^{ \pm \pm}(0)\left(e^{-2 \gamma\left(1+\Phi_{N}^{\mp 2}-\Phi_{N-1}^{\mp 2}\right) t}-1\right)}{\left(\Phi_{N-1}^{\mp 2}-\Phi_{N}^{\mp 2}-1\right)}
\end{aligned}
$$




$$
+\frac{g_{N}^{ \pm 2} \chi_{N, N}^{\mp \mp}(0)\left(e^{-2 \gamma\left(1+\Phi_{N}^{ \pm 2}-\Phi_{N-1}^{\mp 2}\right) t}-1\right)}{\left(\Phi_{N-1}^{\mp 2}-\Phi_{N}^{ \pm 2}-1\right)}, \quad \text { etc. }
$$

Then we take $N$ very large to insure convergence.

To investigate the effect of a cavity damping on the purity and the entanglement, we assume that the qubit-photon system is initially in the state $\rho(0)=J(0)=|\uparrow, \alpha\rangle\langle\uparrow, \alpha|$. Where the qubit is initially in the state $|\uparrow\rangle\langle\uparrow|$, while the field is initially in a coherent state $|\alpha\rangle=\sum_{n=0}^{\infty} q_{n}|n\rangle$, where $q_{n}=e^{-|\alpha|^{2} / 2} \frac{\alpha^{n}}{\sqrt{n !}}$, and $\alpha$ is a complex number in general. With the initial density matrix $J(0)$, the density matrix $\tilde{\rho}(t)$ are found to be

$$
\tilde{\rho}(t)=\sum_{i, j=\uparrow, \downarrow} X^{i j}|i\rangle\langle j|, \quad X^{i j}=\sum_{m, n=0} x_{m, n}^{i j},
$$

where

$$
\begin{aligned}
& x_{m, n}^{\uparrow \uparrow}=\left\{\begin{array}{l}
\Phi_{m}^{+} \Phi_{n}^{+} e^{-i\left(E_{m}^{+}-E_{n}^{+}\right) t} \chi_{m n}^{++}+\Phi_{m}^{-} \Phi_{n}^{-} e^{-i\left(E_{m}^{-}-E_{n}^{-}\right) t} \chi_{m n}^{--} \\
\quad+\Phi_{m}^{+} \Phi_{n}^{-} e^{-i\left(E_{m}^{+}-E_{n}^{-}\right) t} \chi_{m n}^{+-}+\Phi_{m}^{-} \Phi_{n}^{+} e^{-i\left(E_{m}^{-}-E_{n}^{+}\right) t} \chi_{m n}^{-+}, \quad m \neq n ; \\
\Phi_{n}^{+2} \chi_{n}^{+}(t)+\Phi_{n}^{-2} \chi_{n}^{-}(t)+2 q_{n}^{*} q_{n} \Phi_{n}^{2+} \Phi_{n}^{2+} e^{-\gamma(2 n+1) t} \cos \left(2 \eta_{n} t\right), \quad m=n .
\end{array}\right. \\
& x_{m, n}^{\downarrow \downarrow}=\left\{\begin{array}{l}
\Phi_{m}^{-} \Phi_{n}^{-} e^{-i\left(E_{m}^{+}-E_{n}^{+}\right) t} \chi_{m n}^{++}+\Phi_{m}^{+} \Phi_{n}^{+} e^{-i\left(E_{m}^{-}-E_{n}^{-}\right) t} \chi_{m n}^{--} \\
\quad-\Phi_{m}^{-} \Phi_{n}^{+} e^{-i\left(E_{m}^{+}-E_{n}^{-}\right) t} \chi_{m n}^{+-}-\Phi_{m}^{+} \Phi_{n}^{-} e^{-i\left(E_{m}^{-}-E_{n}^{+}\right) t} \chi_{m n}^{-+}, \quad m \neq n ; \\
\Phi_{n}^{-2} \chi_{n}^{+}(t)+\Phi_{n}^{+2} \chi_{n}^{-}(t)-2 q_{n}^{*} q_{n} \Phi_{n}^{-} \Phi_{n}^{-} e^{-\gamma(2 n+1) t} \cos \left(2 \eta_{n} t\right), \quad m=n ; \\
\gamma \int_{0}^{t}\left[\chi_{00}^{++}+\chi_{00}^{--}\right] d \tau \quad m=n=0 .
\end{array}\right. \\
& x_{m, n}^{\uparrow \downarrow}=\left(x_{m, n}^{\downarrow \uparrow}\right)^{\dagger}=\Phi_{m}^{-} \Phi_{n}^{+} e^{-i\left(E_{m}^{+}-E_{n}^{+}\right) t} \chi_{m n}^{++}-\Phi_{m}^{+} \Phi_{n}^{-} e^{-i\left(E_{m}^{-}-E_{n}^{-}\right) t} \chi_{m n}^{--} \\
& +\Phi_{m}^{-} \Phi_{n}^{-} e^{-i\left(E_{m}^{+}-E_{n}^{-}\right) t}-\chi_{m n}^{+-} \Phi_{m}^{+} \Phi_{n}^{+} e^{-i\left(E_{m}^{-}-E_{n}^{+}\right) t} \chi_{m n}^{-+}, \quad \forall m, n .
\end{aligned}
$$

By using the transforms of Eqs. (7), the exact solution of the master equation (21) in the case of a high- $Q$ cavity is given by

$$
\begin{aligned}
\rho(t)= & {\left[X^{\uparrow \uparrow} \cos ^{2} \xi+X^{\downarrow \downarrow} \sin ^{2} \xi-\sin \xi \cos \xi\left(X^{\uparrow \downarrow}+X^{\downarrow \uparrow}\right)\right]|e\rangle\langle e| } \\
& +\left[X^{\downarrow \downarrow} \cos ^{2} \xi+X^{\uparrow \uparrow} \sin ^{2} \xi+\left(X^{\uparrow \downarrow}+X^{\downarrow \uparrow}\right) \sin \xi \cos \xi\right]|g\rangle\langle g| \\
& +\left[X^{\uparrow \downarrow} \cos ^{2} \xi-X^{\downarrow \uparrow} \sin ^{2} \xi+\left(X^{\uparrow \uparrow}-X^{\downarrow \downarrow}\right) \sin \xi \cos \xi\right] \\
& \otimes|e\rangle\langle g|+\left[X^{\downarrow \uparrow} \cos ^{2} \xi-X^{\uparrow \downarrow} \sin ^{2} \xi\right. \\
& \left.+\left(X^{\uparrow \uparrow}-X^{\downarrow \downarrow}\right) \sin \xi \cos \xi\right]|g\rangle\langle e| .
\end{aligned}
$$

In the forthcoming sections we study the dynamical properties of the population inversion, the purity and entanglement for the proposed system of a single-mode microwave cavity field and a superconducting charge qubit. We assume that the SC Cooper-pair box is made from aluminum, with a energy gap of $\sim 2.4 \mathrm{~K}$ (about $50 \mathrm{GHz}$ ) [39]; the charge energy $E_{c h}$ and the Josephson energy $E_{J}$ are $4 E_{c h} / h=149 \mathrm{GHz}$ and $2 E_{J} / h=13.0 \mathrm{GHz}$, respectively. The frequency of the cavity field is taken as $40 \mathrm{GHz}$, corresponding to a wavelength $\sim 0.75 \mathrm{~cm}$. Therefore, the SC energy gap is the largest energy and the quasiparticle 
excitation on the island can be well suppressed at low temperatures. If SQUID area is assumed to be about $50 \mu \mathrm{m} \times 50 \mu \mathrm{m}$, the absolute value $|\lambda|$ of the qubit-photon coupling constant is about $|\lambda|=4 \times 10^{6} \mathrm{rad} \mathrm{s}^{-1}$. The gate voltage is adjusted such that the gate charge is $n_{g}=0.634233$, which can be experimentally achieved [39], then the detuning $2 \delta=9 \times 10^{6} \mathrm{rad} \mathrm{s}^{-1}$ and $\xi \simeq \frac{1}{2} \tan ^{-1}\left(\frac{40}{13}\right) \simeq 36^{\circ}$. In the next section we concentrate on the discussion of the population inversion behaviour.

\section{Population Inversion of the Charge-Qubit}

The junction effects can be used to observe the collapses and revivals phenomena in a condensed matter system. The interaction of the photons with a junction which behaves like an atom undergoing transition between the quantum states of the junction as it absorbs and emits radiation. This in fact would give information about the behaviour of the interaction between the qubit and the electromagnetic field during the time considered. Therefore using above mentioned data we have plotted the function $\left\langle\sigma_{z}(t)\right\rangle$ against the scaled time $10^{6} t$ to display its behaviour for different values of the detuning parameter $\delta$ and decoherence parameter $\gamma$.

In Fig. 1 we plot the population inversion as a function of the scaled time and assuming that the qubit is initially in the excited state and the field in the coherent state with the intensity of the initial coherent parameter to $\alpha=4$, the coupling parameter is taken $\lambda=$ $4 \times 10^{6} \mathrm{rad} \mathrm{s}^{-1}$ related to the junction coupling by Eq. (12). The first case, we consider is the on-resonance case $(\delta=0)$ and without the damping i.e $\gamma=0$. we find that the phenomena
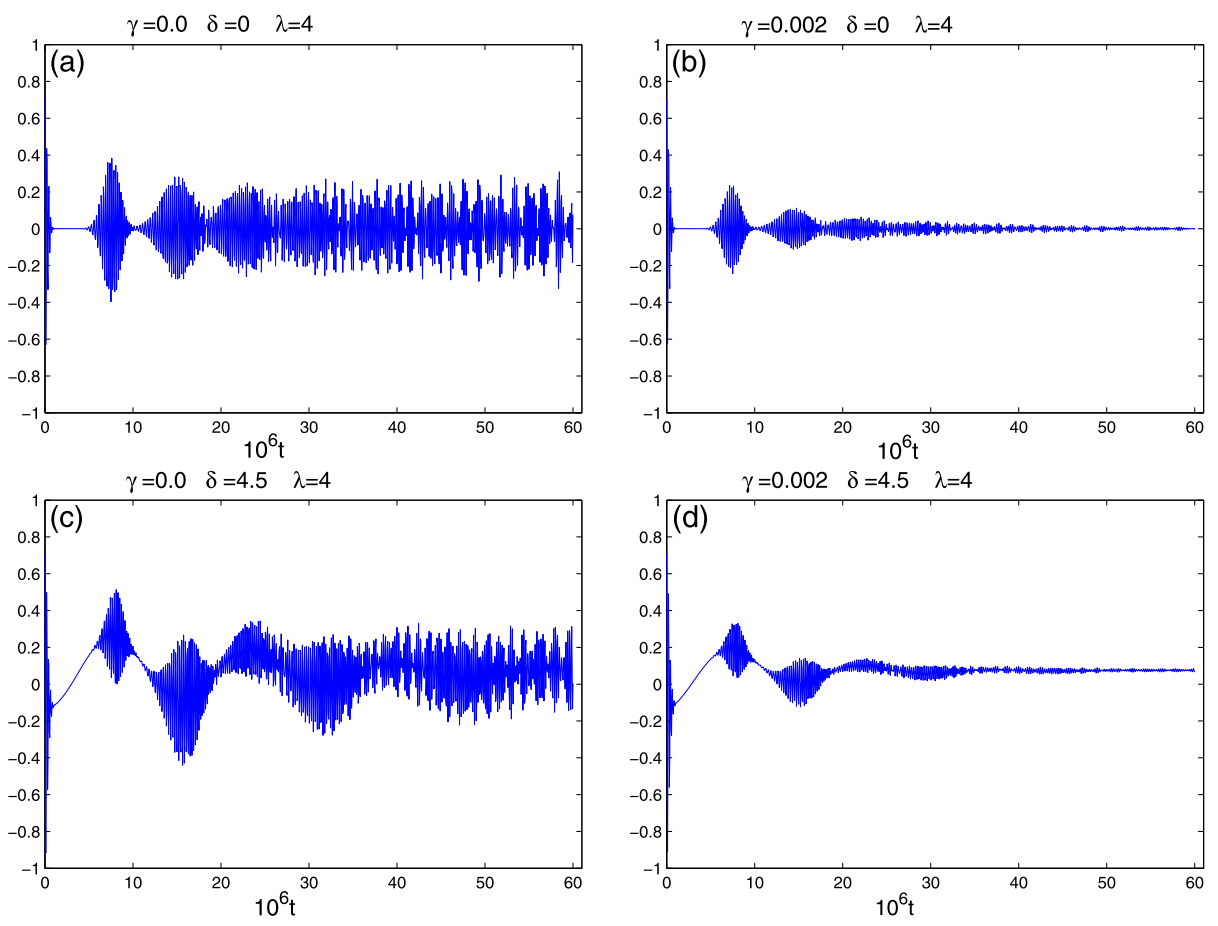

Fig. 1 Time evolution of the population inversion of the charge-Qubit when $|\alpha|^{2}=16$ 
of collapses and revivals are similar to that of the coherent state JCM case. Since the revival times can be estimated as in Refs. [40,41], therefore the revival times for a coherent state can be written as $t_{R}=2 \pi|\alpha|$. We see that the population inversion starts to oscillate showing a long period of collapse after the onset of the interaction then periods of revivals. During this revival periods the function shows fluctuations with a symmetrical behaviour around zero (note that this symmetry becomes from Eq. (10), where the second term vanishes for the on-resonance case) where its extreme occurs between \pm 0.4 . Also, there are short periods of collapse during which the function exhibits slight fluctuations; see Fig. 1(a). When we take the damping into account and adjust $(\gamma=0.002)$, it is observed that the amplitudes of revival oscillations decay to zero as the time develops (see Fig. 1(b)). Which means that the decay of quantum coherence is due to the very specific time evolution described by the master Eq. (3), i.e., due to the amplitude damping. It is remarked that the qubit decays to a mixed state where the probabilities of finding the atom in the excited state and the ground state are equal.

The second case we take the off-resonance case into consideration by adjusting $2 \delta=$ $9 \times 10^{6} \mathrm{rad} \mathrm{s}^{-1}$ and in the absence of the damping parameter $(\gamma=0)$, It is clear from this figure the population inversion shows periodic oscillations between the maximum value $\left\langle\sigma_{z}(t)\right\rangle=0.6$ and minimum value $\left\langle\sigma_{z}(t)\right\rangle=-0.8$ see Fig. 1(c). It is evident from the figures that the collapses region is shifted upward and downward gradually in the inversion curve for the case $\delta \neq 0$. In the meantime, the function shows a small period of revival after the onset of the interaction which is followed with a small period of collapse. When we take the damping into account $(\gamma=0.002)$ and the detuning $\left(2 \delta=9 \times 10^{6} \mathrm{rad} \mathrm{s}^{-1}\right)$, we see that the oscillations of the population function are weakened out and the amplitudes of the revival oscillations decay to zero as the time develops (see Fig. 1(d)). In general the detuning parameter plays two roles: firstly, it weakens the interaction between the atom and the field; secondly, it reduces the transition probability of the atom from the upper level to the mixed state of the stationary case and delays the damping. Furthermore in this case it adds oscillatory behaviour to the form of the collapse and revival due to the mixing of the operators $\sigma_{z}(t)$ and $\sigma_{x}(t)$ as showing in Eq. (10) as observed in [42].

\section{Purity Loss of the States}

There are two sources of purity loss in the present system. One of them is due to the unitary qubit-cavity interaction. This process is usually called entanglement and from the point of view of one of the subsystems, a purity loss will take place. On the other hand, the interaction of the field subsystem with the environment also induces purity loss, and this process is usually called decoherence in the literature. Due to decoherence, a pure state is apt to change into a mixed state. However, in many cases of quantum information processing, one requires a state with high purity and large amount of entanglement.

Here we use the linear entropy as a measure for the purity loss of the states of the global system, of the qubit and cavity, in analogy to what is done for the calculation of the purity in terms of von Neumann (or linear) entropy [43-47] which has similar behavior. The purity loss of the qubit-cavity state can be measured in terms of the linear entropy [48] defined as: $S=1-\operatorname{tr}\left\{\rho^{2}\right\}$. It should be noted that the entropy is a key concept of quantum information [49]. To see what happens to the charge-qubit state, we trace out the cavity variables from the state $\rho(t)$ and get the reduced density matrix: $\rho_{q}=\operatorname{tr}_{f}\{\rho\}$. The linear entropy of the Cooperpair box states can be written as: $S^{q}=1-\operatorname{tr}\left\{\rho_{q}^{2}\right\}$, where $S^{q}$ has a zero value for a pure state. But the reduced density matrix of the cavity state is given by: $\rho_{f}=\operatorname{tr}_{q}\{\rho\}$. The linear entropy 

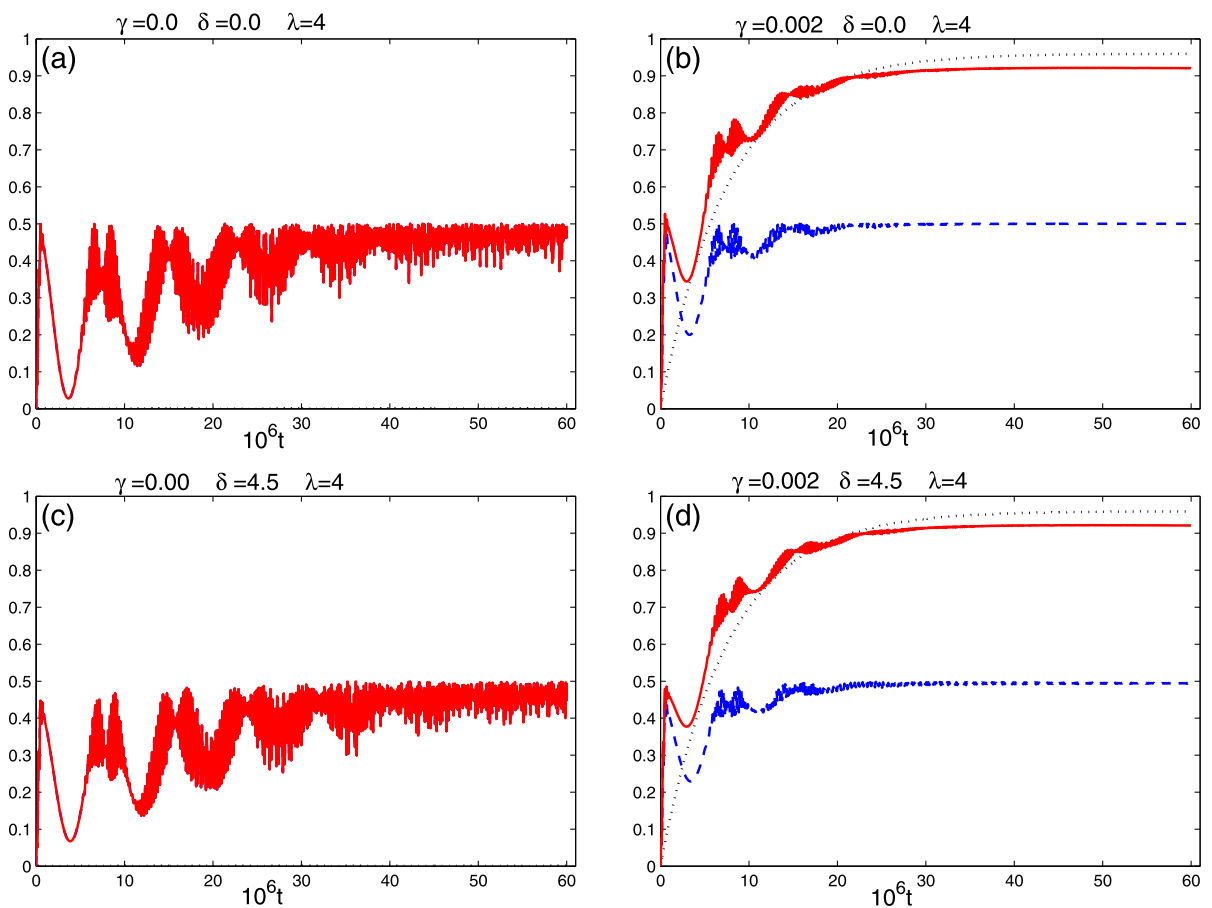

Fig. 2 Dynamics of the mixedness of the global system state (dotted plots), charge-qubite state (dashed dotted plots) and cavity state (solid plots) for $|\alpha|^{2}=16$

of the cavity field state is given by: $S^{f}=1-\operatorname{tr}\left\{\rho_{f}^{2}\right\}$. A necessary and sufficient condition for the ensemble to be described in terms of a pure state is that $\operatorname{Tr}\left[\hat{\rho}_{f}^{2}(t)\right]=1$. For the case $\operatorname{Tr}\left[\rho_{f}^{2}(t)\right]<1$ the field will be in a statistical mixture state. However, for a maximally mixed ensemble corresponds to $\operatorname{Tr}\left[\rho_{f}^{2}(t)\right]=\frac{1}{2}$.

To do the analysis and discuss the purity, we plot the mixedness of the global system state, charge qubit state and cavity state against the dimensionless time $10^{6} t$ assuming that the field is prepared in a coherent state with the initial coherent parameter $\alpha=4$ and the atom in the excited state for different values of the parameters $\delta$ and $\gamma$. In the on-resonance case and absence of the damping $(\gamma=0)$, it is known that $S^{q}=S^{f}$, they have the same time evolution curve. From Fig. 2(a) it is observed that the linear entropy in general satisfies the inequality $0<S^{f}<0.5$ further the system approaches a mixed state showing strong entanglement with regular oscillations at the half of the revival times, while weak entanglement at the half of the collapse times and the system does not reach a pure state at any time $t>0$. In the meantime the maximum value of the linear entropy function becomes nearly 0.5 . This means that the qubit almost reaches the mixed state as verified by the population inversion in Fig. 1(a).

When we take the damping parameter $\gamma$ into account, the linear entropies of the chargequbit and cavity are different. Since the definitions of the linear entropies depend on the offdiagonal terms of the densities (whether total or partial), we must expect that these quantities will be affected by the cavity damping, this is shown distinctively in Fig. 2(b). Owing to amplitude damping, the state is changed from a pure state at $t=0$, to a mixed state as time develops. We note that the total entropy increases monotonically and no longer equals 
to zero while $S^{q}$ and $S^{f}$ are no longer equal and hence cannot be used as a measure for entanglement. But they may be used to study the purity loss. After a long time, the purity loss of the full system $S$ approximately follows that for the linear entropy of the cavity and the time scales of the cavity and the full systems decoherence are the same. It is remarked that the field attains higher entropy than the charge-qubit, so the purity loss of the field state is faster than the qubit purity loss as observed in Fig. 2(b).

When we take the off-resonance case into consideration by setting $2 \delta=9 \times 10^{6} \mathrm{rad} \mathrm{s}^{-1}$ and in absence of the damping parameter $(\gamma=0)$. We see that the linear entropy has the same behaviour excepting the short time at the beginning interaction the extreme (minimum) of function $S^{q}$ decreases and more fluctuations can be seen, however, with some interference between patterns see Fig. 2(c). This means that the field becomes in a mixture state and never reaching its maximal. As we add the damping parameter $\gamma=0.002$ into consideration, the field entropy separates further from the charge-qubit entropy, the amplitudes of the fast oscillations diminish and the minima (maxima) at half-revival times as compared with the previous case see Figs. 2(b) and 2(c). We also see a more rapid suppression of quantum coherences and increase of the values of the entropies $S$ and $S^{f}$, while $S^{q}$ converges to 0.5 as $t$ develops (see Fig. 2(d)).

\section{Entanglement and Total Correlation}

We now consider the influence of decoherence on quantum entanglement of the charge qubit-cavity system under consideration. Since the interaction of the system with environment makes the qubit-cavity pure state evolve to a mixed state, the linear entropy is not a better measure for the entanglement of the mixed state. A good measure to describe the amount of entanglement is the negativity, $N(\rho)=\max \left(0,-\sum_{j} \lambda_{j}\right)$, where $\lambda_{j}$ are negative eigenvalues of the partially transposed density matrix of the qubit-cavity [50]. For an entangled mixed state, the negativity is positive whereas it vanishes for unentangled states.

For the environment, not only entanglement but also classical correlation of the system and environment can make the system evolve from the initial pure state into a mixed one. So we will also look at the total correlation between the charge-qubit and the field as quantified by the mutual information $M(\rho)$ [24], which is defined as: $M(\rho)=\frac{1}{2}\left[S^{q}+S^{f}-S\right]$. The mutual entropy $M(\rho)$ as defined above is positive, and is zero if and only if the marginal states are not correlated. It is a measure of both quantum and classical correlation residing in the composite system. We will compare the two quantities $N(\rho)$ and $M(\rho)$ (i.e., the amount of entanglement and the mutual information) to understand how much the purely quantum correlations contribute to the total correlations.

For the on-resonance case and in absence of the damping parameter, we see that the $M(\rho)$ and $N(\rho)$ follows the same behaviour for the time interval considered with the values of the extreme are different as observed in Fig. 3(a). Also again we observed that strong entanglement occurs between the qubit and the cavity at half the revival times while at the half of collapse times the system shows weak entanglement see Figs. 1(a) and 3(a). When we take the damping into account $(\gamma=0.002)$, it is to be observed that as time develops the negativity of both $N(\rho)$ and $M(\rho)$ becomes smaller but they differ in the amplitudes, however they have the same trend in general see Fig. 3(b). When we take the off-resonance case into consideration by setting $2 \delta=9 \times 10^{6} \mathrm{rad} \mathrm{s}^{-1}$ and $(\gamma=0)$, we see that the functions $M(\rho)$ and $N(\rho)$ follow same behaviour except the short time at the beginning of interaction the extreme (minimum) of function $S^{q}$ decreases and more fluctuations can be seen, however, with some interference between patterns see Fig. 3(c). After adding the damping, 

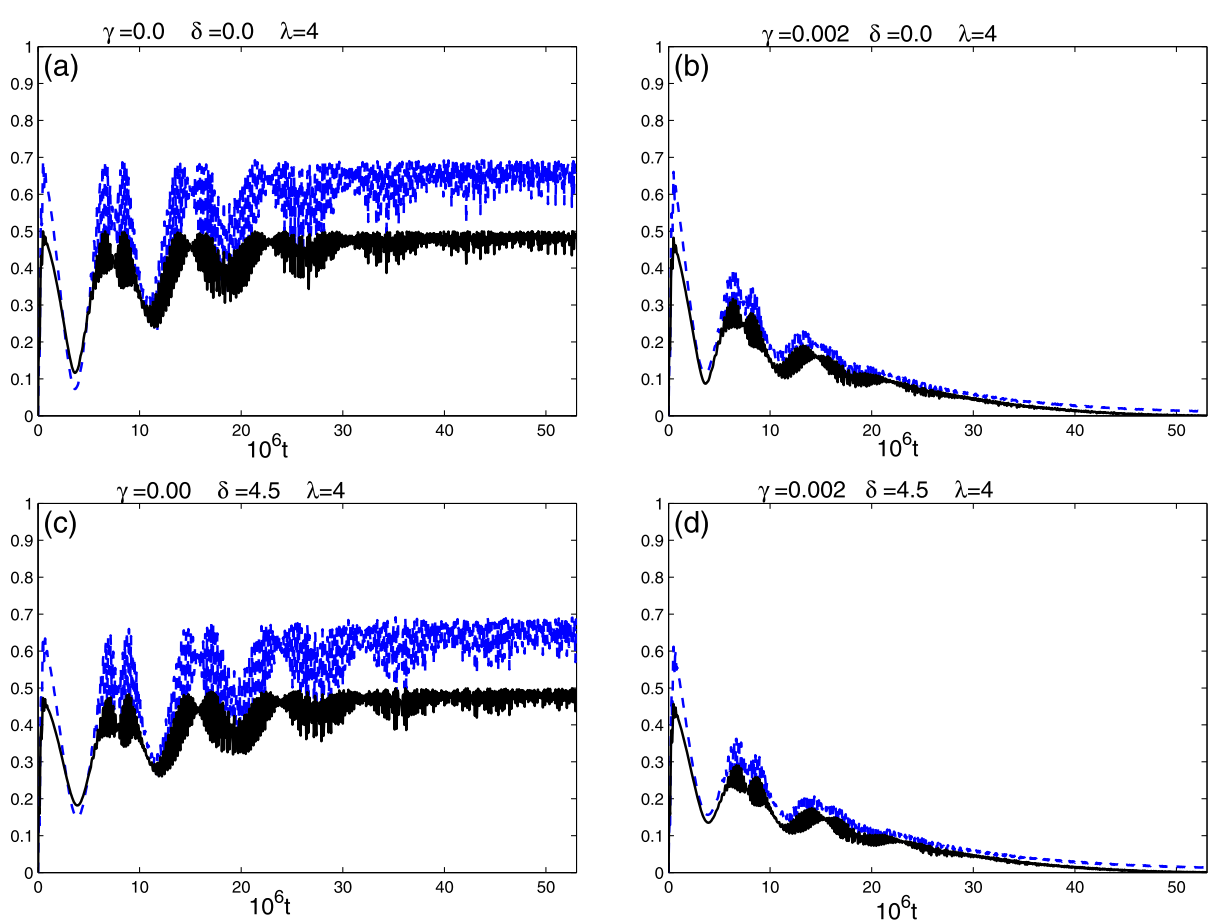

Fig. 3 Dynamics of the negativity of the global system state (solid plots) and its total correlation (dashed plots) for $|\alpha|^{2}=16$

we observe that the entanglement between the two subsystems becomes weaker and the amplitudes of local maxima and minima are further reduced as observed in Fig. 3(d). During the repeated periods of entanglement and disentanglement, the qubit and field lose and gain their coherence but the coherence recovered by the qubit never equal to what lost. The field finally goes into a pure state (the vacuum state) and its coherence is lost completely. We note that $M(\rho)$ and $N(\rho)$ have a very strong sensitivity to the damping parameter $\gamma$, where with the increase of $\gamma$, the values of both $M(\rho)$ and $N(\rho)$ approach zero faster.

\section{Conclusions}

A general interaction of a single-mode microwave cavity field with a superconducting charge qubit is investigated. Applying a canonical transformation to the qubit system, we have managed to transform the model to the usual JCM. The analytical solution for the master equation of the system is obtained. The population inversion in absence and presence of both the damping and the detuning parameters is discussed. It is found that by increasing the damping parameter the purity of the system and environment is lost. The results show that with an increase of the damping parameter the entanglement between the subsystem increases to maximum as the time increases. It should be noted that the problem we have considered in the present communication is regarded as a generalization of that considered in Ref. [51] where the entanglement is discussed in absence of the damping factor.

Acknowledgement MSA extends his appreciation to the Deanship of Scientific Research at KSU for funding the work through the research group project No. RGP/VPP/101. 
Open Access This article is distributed under the terms of the Creative Commons Attribution License which permits any use, distribution, and reproduction in any medium, provided the original author(s) and the source are credited.

\section{References}

1. Makhlin, Y., Schön, G., Shnirman, A.: Rev. Mod. Phys. 73, 357 (2001)

2. Clarke, J., Wilhelm, F.K.: Nature (London) 453, 1031 (2008)

3. Chen, M.-Y., Tu, M.W.Y., Zhang, W.-M.: Phys. Rev. B 80, 214538 (2009)

4. Zagoskin, A.M., Grajcar, M., Omelyanchouk, A.N.: Phys. Rev. A 70, 060301 (2004)

5. You, J.Q., Tsai, J.S., Nori, F.: Phys. Rev. Lett. 89, 197902 (2002)

6. You, J.Q., Nori, F.: Phys. Rev. B 68, 064509 (2003)

7. Blais, A., van den Brink, A.M., Zagoskin, A.M.: Phys. Rev. Lett. 90, 127901 (2003)

8. Liu, Y.-X., Sun, C.P., Nori, F.: Phys. Rev. A 74, 052321 (2006)

9. You, J.Q., Nori, F.: Phys. Today 58, 42 (2005)

10. Averin, D.V.: Fortschr. Phys. 48, 1055 (2000)

11. Martinis, J.M., Nam, S., Aumentado, J., Urbina, C.: Phys. Rev. Lett. 89, 117901 (2002)

12. Benatti, F., Floreanini, R., Realpe-Gomez, J.: J. Phys. A, Math. Theor. 41, 235304 (2008)

13. You, J.Q., Nori, F.: Phys. Rev. B 68, 064509 (2003)

14. Bennett, C.H., Di Vincenzo, D.P., Smolin, J.A., Wootters, W.K.: Phys. Rev. A 54, 3824 (1996)

15. Horodecki, M.: Quantum Inf. Comput. 1, 3 (2001)

16. Wootters, W.K.: Quantum Inf. Comput. 1, 27 (2001)

17. Vedral, V.: Rev. Mod. Phys. 74, 197 (2002)

18. Horodecki, R., Horodecki, P., Horodecki, M., Horodecki, K.: Rev. Mod. Phys. 81, 865 (2009)

19. Nielsen, M.A., Chuang, I.L.: Quantum Computation and Quantum Information. Cambridge University Press, Cambridge (2000)

20. Ikram, M., Li, F.L., Zubairy, M.S.: Phys. Rev. A 75, 062336 (2007)

21. Guo, J.L., Song, H.S.: Eur. Phys. J. D 61, 791 (2011)

22. Yu, T., Eberly, J.H.: Phys. Rev. B 68, 165322 (2003)

23. Plenio, M.B., Vedral, V.: Phys. Rev. A 57, 1619 (1998)

24. Barnett, S.M., Phoenix, S.J.D.: Phys. Rev. A 40, 2404 (1989)

25. Vidal, G., Werner, R.F.: Phys. Rev. A 65, 032314 (2002)

26. Schumacher, B., Westmoreland, M.D.: Phys. Rev. A 74, 042305 (2006)

27. Groisman, B., Popescu, S., Winter, A.: Phys. Rev. A 72, 032317 (2005)

28. Henderson, L., Vedral, V.: J. Phys. A, Math. Gen. 34, 6899 (2001)

29. Vedral, V.: Phys. Rev. Lett. 90, 050401 (2003)

30. Yang, D., Horodecki, M., Wang, Z.D.: Phys. Rev. Lett. 101, 140501 (2008)

31. Liu, Y.-X., Wei, L.F., Nori, F.: Europhys. Lett. 67, 941 (2004)

32. Liu, Y.-X., Wei, L.F., Nori, F.: Phys. Rev. A 71, 063820 (2005)

33. Nakamura, Y., Pashkin, Y.A., Tsai, J.S.: Nature (London) 398, 786 (1999)

34. Nakamura, Y., Pashkin, Y.A., Tsai, J.S.: Phys. Rev. Lett. 87, 246601 (2001)

35. Pashkin, Y.A., Yamamoto, T., Astafiev, O., Nakamura, Y., Averin, D.V., Tsai, J.S.: Nature (London) 421, 823 (2003)

36. Barnett, S.M., Knight, P.L.: Phys. Rev. A 33, 2444 (1986)

37. Puri, R.R., Agarwal, G.S.: Phys. Rev. A 35, 3433 (1987)

38. Puri, R.R., Agarwal, G.S.: Phys. Rev. A 39, 3879 (1988)

39. Lehnert, K.W., Bladh, K., Spietz, L.F., Gunnarson, D., Schuster, D.I., Delsing, P., Schoelkopf, R.J.: Phys. Rev. Lett. 90, 027002 (2003)

40. Eberly, J.H., Narozhny, N.B., Sanchez-Mondragan, J.J.: Phys. Rev. Lett. 44, 1323 (1980)

41. Narozhny, N.B., Sanchez-Mondragan, J.J., Eberly, J.A.: Phys. Rev. A 23, 2361 (1981)

42. Abdalla, M.S., Khalil, E.M., Obada, A.-S.F.: Ann. Phys. 326, 2486 (2011)

43. Santos, E., Ferrero, M.: Phys. Rev. A 62, 024101 (2000)

44. Bennett, C.H., Bernstein, H.J., Popescu, S., Schumacher, B.: Phys. Rev. A 53, 2046 (1996)

45. Munro, W.J., James, D.F.V., White, A.G., Kwiat, P.G.: Phys. Rev. A 64, 030302 (2001)

46. Peixoto, J.G., Nemes, M.C.: Phys. Rev. A 59, 3918 (1999)

47. Obada, A.-S.F., Hessian, H.A., Mohamed, A.-B.A.: J. Phys. B, At. Mol. Opt. Phys. 40, 2241 (2007)

48. Pters, N.A., Wei, T.C., Kwiat, P.G.: Phys. Rev. A 70, 052309 (2004)

49. Abdalla, M.S., Eleuch, H., Peřina, J.: J. Opt. Soc. Am. B 29(4) (2012)

50. Peres, A.: Phys. Rev. Lett. 77, 1413 (1996)

51. Abdalla, M.S., Obada, A.-S.F., Khalil, E.M., Mohamed, A.-B.A.: Prog. Theor. Exp. Phys. (2013). doi:10. 1093/ptep/ptt056. 083A01 (14 pages) 